\title{
A Restraint-Free Small Animal SPECT Imaging System With Motion Tracking
}

\author{
A. G. Weisenberger, S. S. Gleason, J. Goddard, B. Kross, S. Majewski, S. R. Meikle, Senior Member, IEEE, \\ M. J. Paulus, M. Pomper, V. Popov, M. F. Smith, Member, IEEE, B. L. Welch, and R. Wojcik
}

\begin{abstract}
We report on an approach toward the development of a high-resolution single photon emission computed tomography (SPECT) system to image the biodistribution of radiolabeled tracers such as Tc-99m and I-125 in unrestrained/unanesthetized mice. An infrared (IR)-based position tracking apparatus has been developed and integrated into a SPECT gantry. The tracking system is designed to measure the spatial position of a mouse's head at a rate of 10-15 frames per second with submillimeter accuracy. The high-resolution, gamma imaging detectors are based on pixellated $\mathrm{NaI}(\mathrm{Tl})$ crystal scintillator arrays, position-sensitive photomultiplier tubes, and novel readout circuitry requiring fewer analog-digital converter (ADC) channels while retaining high spatial resolution. Two SPECT gamma camera detector heads based upon position-sensitive photomultiplier tubes have been built and installed onto the gantry. The IR landmark-based pose measurement and tracking system is under development to provide animal position data during a SPECT scan. The animal position and orientation data acquired by the tracking system will be used for motion correction during the tomographic image reconstruction.
\end{abstract}

Index Terms-Motion tracking, multimodality imaging, small animal single photon emission computed tomography (SPECT).

\section{INTRODUCTION}

$\mathbf{F}$ OR nearly 15 years, researchers have used single photon emission computed tomography (SPECT) systems for animal studies. Much of the early work focused on the use of clinical systems fitted with pinhole collimators. In 1991, a group at Massachusetts General Hospital [1], Boston, MA, used a clinical gamma scanner configured with pinhole collimators to study blood flow in rats and hamsters. Weber et al. [2] and Jaszczak, et al. [3] describe a pinhole SPECT system using a single-pinhole collimator attached to a clinical single-head SPECT scanner. Ishizu et al. [4] report using four pinhole collimators attached to a standard clinical four-head SPECT

Manuscript received November 19, 2003. The Southeastern Universities Research Association (SURA) operates the Thomas Jefferson National Accelerator Facility (Jefferson Laboratory) for the U.S. Department of Energy (DOE) under Contract DE-AC05-84ER40150. This work is supported in part by the DOE Office of Biological and Environmental Research in the Office of Science through the DOE Medical Imaging program and from the DOE Office of Nuclear Physics.

A. G. Weisenberger, B. Kross, S. Majewski, V. Popov, M. F. Smith, B. L. Welch, and R. Wojcik are with the Jefferson Laboratory, Newport News, VA 23606 USA (e-mail: drew @jlab.org).

S. S. Gleason, J. Goddard, and M. J. Paulus are with the Oak Ridge National Laboratory, Oak Ridge, TN 37831 USA (e-mail: gleasonss@ornl.gov).

S. R. Meikle is with the Royal Prince Alfred Hospital, Camperdown, NSW 2050, Australia (e-mail: steve@ cs.usyd.edu.au).

M. Pomper is with The Johns Hopkins University School of Medicine, Baltimore, MD 21218 USA (e-mail: mpomper@jhmi.edu).

Digital Object Identifier 10.1109/TNS.2005.851399 scanner. Since these early works were presented, numerous researchers have developed systems with increasing resolution and sensitivity. The maturity and availability of SPECT tracer compounds has facilitated the acceptance of this technology. In research hospitals where clinical SPECT systems are readily available, pinhole collimation provides an excellent means for obtaining the requisite resolution for laboratory animal studies.

For researchers without access to clinical systems, however, there has been interest to develop dedicated animal systems. One of the first to do this was Dilmanian et al. [5] who reported on the development of a high-resolution micro-channel-platebased SPECT system to image I- 125 in rats and mice with a spatial resolution of 1-mm full-width at half-maximum (FWHM). Development of small animal systems was aided significantly by the development of position-sensitive photomultiplier tubes (PSPMTs), and virtually all dedicated animal SPECT systems employ some form of PSPMT. We have constructed imaging systems based upon PSPMTs coupled to pixillated scintillator arrays for small animal studies [6], [7]. Schramm reported an animal SPECT apparatus which used a single large area PSPMT, a continuous $\mathrm{NaI}(\mathrm{Tl})$ crystal, and a parallel hole collimator [8]. In 2001, a group at the National Institutes of Health [9], Bethesda, MD, demonstrated the use of a PSPMT-based system for both SPECT and positron emission tomography (PET) studies of laboratory animals.

The success of these laboratory programs precipitated the development of the first dedicated commercial laboratory animal SPECT system [10], [11] that employs PSPMTs coupled to arrays of pixilated scintillator crystals and pinhole collimators.

Researchers have acquired in vivo images of transporter and receptor distributions in small animal models such as rats and mice [12], [13], and have also focused specifically on the brain using PET and SPECT [14], [15]. These studies are limited, however, by the necessity of using anesthetic or physical restraint during image acquisition. Both methods of restraint have the potential to alter the neurological and physiological processes being studied [16]. Two groups have developed surgically implanted scintillator-based probes to measure the uptake of radiolabeled tracers in the vicinity of the implanted intracerebral microprobes for anesthetized and unanesthetized rodent studies [17], [18]. Others are pursuing a PET-based system to image the brain of an unanesthetized rat by use of a miniaturized PET detector intended for attachment to a rat's head that requires a counter balance to offset the weight of the detector [19].

All of these techniques require a potentially intrusive manipulation of the animal. Oak Ridge National Laboratory (ORNL), 


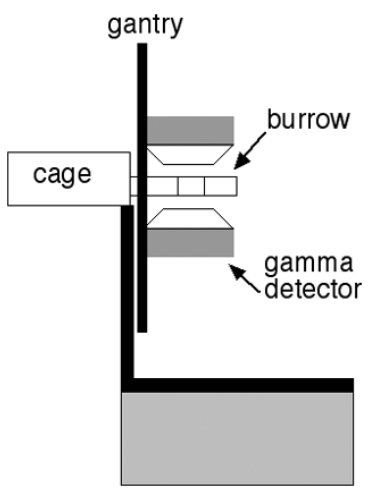

Fig. 1. Diagram of gantry layout.

Oak Ridge, TN, and Thomas Jefferson National Accelerator Facility (JLab), Newport News, VA, in collaboration with Royal Prince Alfred Hospital, Sydney, Australia, and The Johns Hopkins University, Baltimore, MD, are developing a new methodology for imaging unrestrained animals. With this methodology the animal position is recorded during gamma-ray image acquisition and the gamma-ray data is reconstructed into a fixed reference frame taking into account the time-varying animal orientation. Our goal is to develop a system to acquire high-resolution SPECT images of the head region of an unrestrained, unanesthetized mouse and to register these image volumes with previously acquired microCT data sets of the same mouse. The animal will be anesthetized during the microCT data acquisition. The obtained microCT images will serve as a reference frame for the SPECT data. An infrared (IR)-based tracking system provides the position and pose of the mouse's head during the imaging session.

The purpose of this paper is to describe the approach, design, and construction of a SPECT based with an integrated animal three-dimensional (3D) pose measurement system. To illustrate the various components we will provide examples of the operational performance of the different subsystems.

\section{IMAGING CONCEPT}

Two major systems are involved in our imaging concept: 1) a microCT system to obtain an anatomical image of the animal and 2) a specialized SPECT gantry equipped with an animal tracking system. The ORNL microCT scanners have been in operation for approximately five years and have been used for many studies including secondary (AA) amyloidosis models, tumor models, obesity models, models of skeletal abnormalities, and a number of mutant strains [20]-[22].

For this project we have designed and built a SPECT gantry that has an IR-based tracking system integrated to it. The gantry has been constructed in which at the center of rotation is a $3-\mathrm{cm}$ diameter cylindrical mouse burrow (see Fig. 1). Unrestrained mice will spend extended periods of time in small tubes that serve as burrows [23].

We are developing an imaging system in which the gamma camera image acquisition, the IR optical tracking system, and the gantry motion are each controlled by a separate desktop computer. Gamma event data, animal tracking data, and gantry

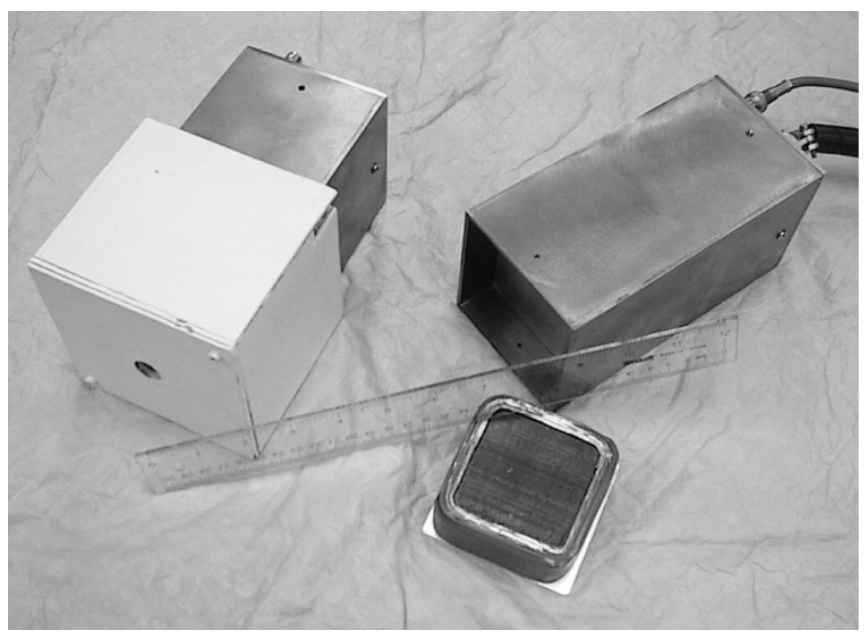

Fig. 2. Photograph of the detector heads in tungsten bodies. The detector on the left is fitted with the white pinhole fixture. The detector head on the right has the parallel hole collimator removed.

position data are all stored on their respective computers each with a time stamp obtained from a common external clock. The data files are later used to reconstruct SPECT images to the small animal reference frame (SARF) based on a previously obtained microCT of the animal. The IR tracking system consists of two high-speed complementary metal-oxide-semiconductor (CMOS) cameras that are mounted horizontally on a platform facing the burrow. Infrared light-emitting diode (LED) arrays and beam splitters are mounted in front of the cameras to illuminate the animal.

\section{SPECT SYSTEM}

Two gamma cameras based on PSPMTs were constructed. The detector heads are placed opposite each other on the gantry. One is equipped with a parallel hole collimator and the other with a pinhole collimator.

The SPECT detector heads are based on the Hamamatsu R2487 PSPMT. The R2487 is a crossed-wire anode PSPMT that has $18 \mathrm{X}$ and $17 \mathrm{Y}$ anodes and an active area of $7.5 \mathrm{~cm} \times 7.5$ $\mathrm{cm}$. The PSPMTs are coupled to $\mathrm{NaI}(\mathrm{Tl})$ arrays obtained from Saint Gobain (Newbury, OH) in which the crystal elements are $1 \mathrm{~mm} \times 1 \mathrm{~mm} \times 5 \mathrm{~mm}$ in size. There is a $0.25-\mathrm{mm}$ white diffuse reflecting glue septum between each element. The energy resolution for Tc $99 \mathrm{~m}$ emissions is $13 \%$.

The detector head housing was constructed of tungsten to shield the $140-\mathrm{keV}$ gamma-ray emissions of Tc99m. The parallel collimator is lead foil, hexagonal holes in a hexagonal array in which the openings are $1.1 \mathrm{~mm}$ flat-to-flat in size. The septa are $0.17 \mathrm{~mm}$ thick and the collimator thickness is $24.5 \mathrm{~mm}$ resulting in a spatial resolution of $\sim 2.4-\mathrm{mm}$ FWHM at a source to detector distance of $2 \mathrm{~cm}$. The sensitivity of the system with the parallel hole collimator is $280 \mathrm{cpc} / \mu \mathrm{Ci}$. See Fig. 2 for a photograph of the R2487-based detector heads.

The readout circuitry was accomplished by making use of a subtractive resistive readout of the crossed-wire anodes which we have described elsewhere [24]. The 18X and 17Y anodes were connected together in a subtractive readout fashion resulting in four channels to readout per detector head. 


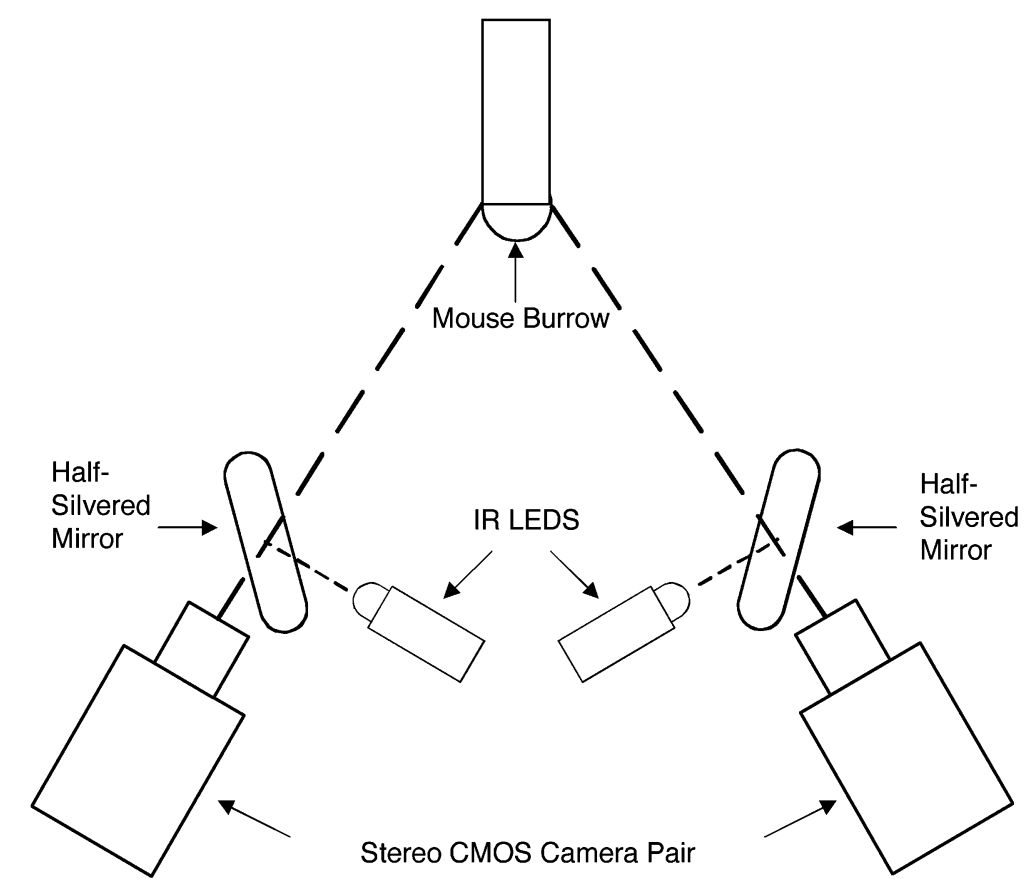

Fig. 3. Schematic of tracking system. The placement of the stereo camera pair is shown in which the mouse with retroreflectors is illuminated by IR LEDs through half-silvered mirrors.

The data acquisition system for the gamma cameras consists of one 16 channel ADC card (Datel, Inc., Mansfield, MA), a discriminator circuit, a millisecond clock to time-stamp the gamma-ray data, and a 10-ms clock used to synchronize the gamma-ray data with the mouse motion data. This Datel (model PCI-416L) PCI computer card is a 16-channel, 12-bit simultaneous sample-and-hold ADC card with 5-V input amplitude range. The 16-channel ADC cards and the millisecond clock are located in a PC that is used to control the gamma camera data acquisition system. The discriminator circuit and $10 \mathrm{~ms}$ clock are external to the PC. For every triggered event, a 12-bit digital signal is transferred to system memory for each ADC channel two at a time, in a 32-bit word. The raw gamma-ray data, the calculated position and energy information, the millisecond clock data, and the 10-ms data are all saved within the event data file for postprocessing and analysis with the IR acquired mouse motion data. The KmaxNT development software package from Sparrow Corporation (Daytona Beach, FL) was used to develop software to control the acquisition. This control software generates an image by processing the raw anode data on an event-byevent basis. The data acquisition system and event structure in described in more detail in [25].

\section{Mouse Position Tracking System}

In clinical nuclear medicine imaging, the implementation of position tracking has been pursued to reduce the affect of patient movement on image quality. Position tracking using a commercial optical system for three dimensional position and orientation measurements requiring a head apparatus has been reported [26], [27]. Others have developed systems to perform optical tracking of surface motion without rotation using a stretchable garment with color patches [28]. Tracking using a commercial mechanical tracking system unsuitable for automated measurements has been reported [29]. In addition, several groups have worked on motion correction using SPECT data without an external position measurement system [30]-[32].

In our approach, the tracking system is based on IR imaging of extrinsic markers and is composed of two high-speed infrared cameras mounted horizontally on a platform facing the tubular burrow in which the animal is unrestrained while being imaged [33]. The system is designed to measure the position of a mouse's head at a rate of 10-15 frames per second with submillimeter accuracy. Infrared LED arrays and beam splitters are mounted in front of the cameras to coaxially illuminate at least three hemispherical retroreflective markers placed on the mouse's head. The markers are spherical with a small flat spot on one side so that it is suitable for attaching to the body. Fig. 3 is a diagram illustrating the placement of the IR LEDs and stereo camera pair.

Two cameras in a stereo approach are used to measure 3D point locations of markers located on the animal's head. The markers are retroreflective and require that the IR illumination be directed parallel along the optical axis of the camera. Since the IR markers are retroreflective, the light has to travel along (and parallel to) the camera's optical axis so that the reflected light will reflect directly back through the camera's optics. The retroreflective markers appear as bright, nonuniform areas in the acquired image. Segmentation of these markers and calculation of their centroids is accomplished in two steps. First, a conservative threshold is applied to the image to ensure that at least the brightest portion of each marker is identified. Second, these segmented pixels are used as seed points for a two-dimensional (2D) region-growing algorithm that contiguously expands the marker areas to cover the entire marker area. The centroid is then calculated for each of the connected regions. The approach 
for accurate measurement requires that both intrinsic and extrinsic calibration be performed for each camera [34]. Finally, the coordinate transformation between cameras is calculated.

\section{OPERATIONAL EXAMPLES}

The gantry at ORNL with the IR-based animal tracking system was equipped with the two gamma cameras. Initial tests of the gantry system in SPECT mode of operation using the gamma cameras has been completed using phantoms and mice. The IR-based optical system using extrinsic IR markers has been tested with phantoms and live mice. The development and testing of the software for the merging of the gamma-camera-based imaging data with the IR tracking data has been achieved with phantoms.

\section{A. SPECT Operation}

The detector heads were used to develop and test the IR tracking system and to perform SPECT studies to validate the gantry and SPECT projection acquisition. No SPECT reconstruction has yet been done of any moving object or mouse. We have used the SPECT system to obtain SPECT reconstruction of immobile mice. An example of a I-125 labeled antibody SPECT imaging study using a dead mouse is shown in Fig. 4. Shown is a merged image of a microCT and reconstructed slice.

\section{B. Gamma Planar Imaging With IR Position Correction}

The accuracy of the tracking system was tested using three IR reflectors on a plastic phantom. By using a micrometer measurement stage we tested motion tracking accuracy in all six degrees of freedom: axially (along the axis of system rotation), transaxially in $x$ and $y$ (horizontal and vertical relative to axis of system rotation), and angular motion (roll, pitch, and yaw). The results are shown in Table I.

Fig. 5 is an example of a test of the software to do a simple motion correction. In this example we obtained a set of planar images in which we tracked a moving Americium 241 calibration source. The linear motion in one plane of a 2-mm-diameter point source in a plastic tube with IR retroreflectors attached was tracked while simultaneously collecting a gamma planar image. The source was placed $\sim 2 \mathrm{~cm}$ in front of the detector face. Time-correlated list mode data containing the tracking coordinates and the gamma imaging data acquired from the gamma cameras equipped with the parallel hole collimator were obtained of a moving radioactive phantom. The gamma camera spatial resolution for this geometry was $\sim 2.4-\mathrm{mm}$ FWHM. In the left panel in Fig. 5(a) is an image of the phantom acquired at two locations separated by $10 \mathrm{~mm}$. In the left panel in Fig. 6(a) is an image of the phantom acquired while the phantom was wiggled back and forth over a distance of $12 \mathrm{~mm}$.

The top-right panels of Figs. 5(a) and 6(a) are the corrected images obtained by mapping the imaging data based on the tracking information. Gamma camera and IR tracking data were taken continuously in list mode and then postprocessed. Vertical line profiles are shown in Figs. 5(b) and 6(b). The motion correction accuracy clearly exceeds the spatial resolution of the gamma camera for the source distance in this example.

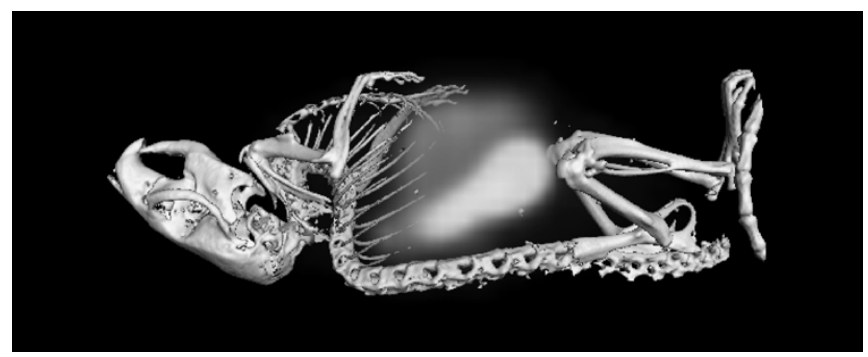

Fig. 4. Merged CT and SPECT image of dead mouse with a I-125 labeled Serum amyloid P component (SAP). The specimen was obtained from Wall at the University of Tennessee.

TABLE I

TRACKING SYSTEM ACCURACY

\begin{tabular}{c|c|c}
\hline Direction & $\begin{array}{c}\text { Actual } \\
\text { Distance }\end{array}$ & Measured \\
\hline Translational (mm) & & \\
\hline $\mathrm{Z}$ (axial) & 12.7 & 12.73 \\
\hline $\mathrm{X}$ (transaxial) & 10 & 10.02 \\
\hline Y (transaxial) & 10 & 9.94 \\
\hline Orientation (degrees) & & \\
\hline roll & 6 & 5.6 \\
\hline pitch & 5 & 5.5 \\
\hline yaw & 6 & 5.8 \\
\hline
\end{tabular}

\section{Live Animal Test of Tracking Concept}

The tracking system has been tested with a live mouse, and the position coordinates of a mouse's head was successfully tracked (see Fig. 7). In these tests, a set of three retroreflective markers are glued to the upper portion of the head: two above the eyes and one near the end of the nose. The mouse was then placed in a 42-mm-diameter clear Pyrex glass tube, one end of which is enclosed and rounded for viewing by the tracking cameras.

In the example shown in Fig. 7, the mouse was tracked continuously for approximately one minute. In the right panel of Fig. 6, the three intense regions are the images of the IR retroreflectors from one of the two IR CMOS cameras. The numbers and outlines show the result of the tracking segmentation algorithm acquiring the moving IR illuminated markers.

\section{DisCUSSION AND FUtURE DiReCTIONS}

We have demonstrated that our imaging approach of tracking the head position and the acquisition of gamma projection images of an unrestrained/unanesthetized mouse is promising. The use of three separate computers generating three different list mode time-tagged gamma, gantry orientation, and mouse position data to image a moving phantom was shown to work. We are pursuing SPECT phantom studies with a moving phantom. No SPECT reconstruction has yet been done of any moving object. The $5 \mathrm{~cm} \times 5 \mathrm{~cm}$ active areas of the gamma cameras are too small to image a whole mouse and accommodate larger animal motion. To address this we have begun replacement of the existing gamma cameras with larger detector heads. We are also investigating an additional method of tracking the mouse that does not require the attachment of extrinsic markers on the animal and uses intrinsic features of the mouse's anatomy. 

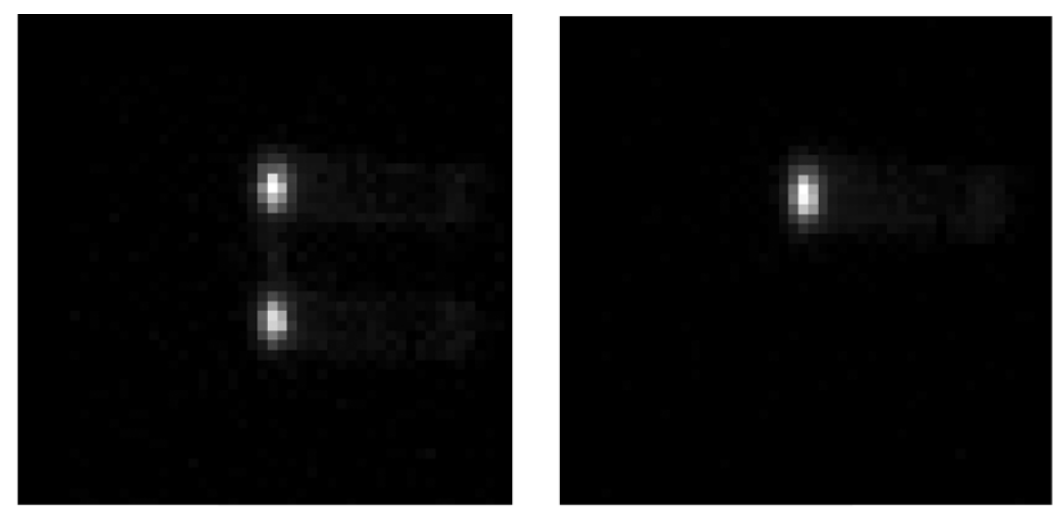

(a)
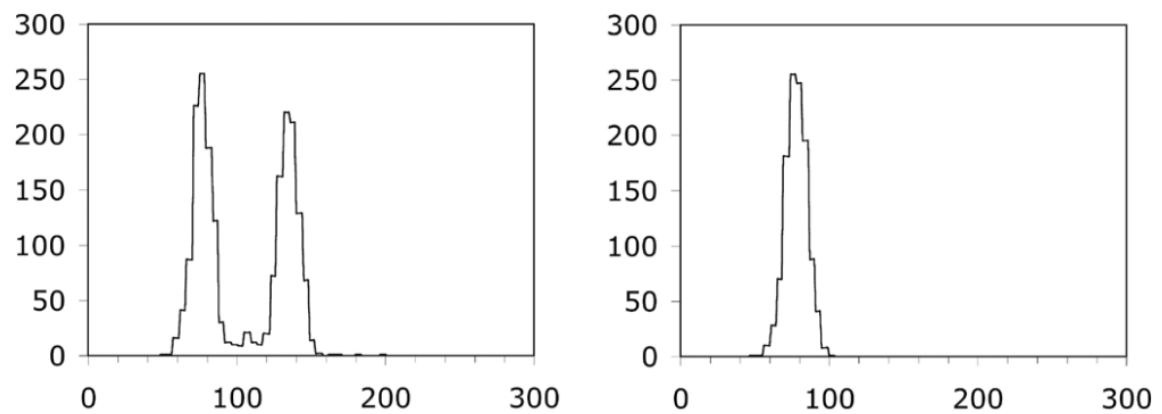

(b)

Fig. 5. (a) Planar images of Americium 241 point source moved to different locations while being imaged by the gamma cameras and the IR tracking system. (b) Profiles (in arbitrary units) are of a vertical line through the center of the source image.
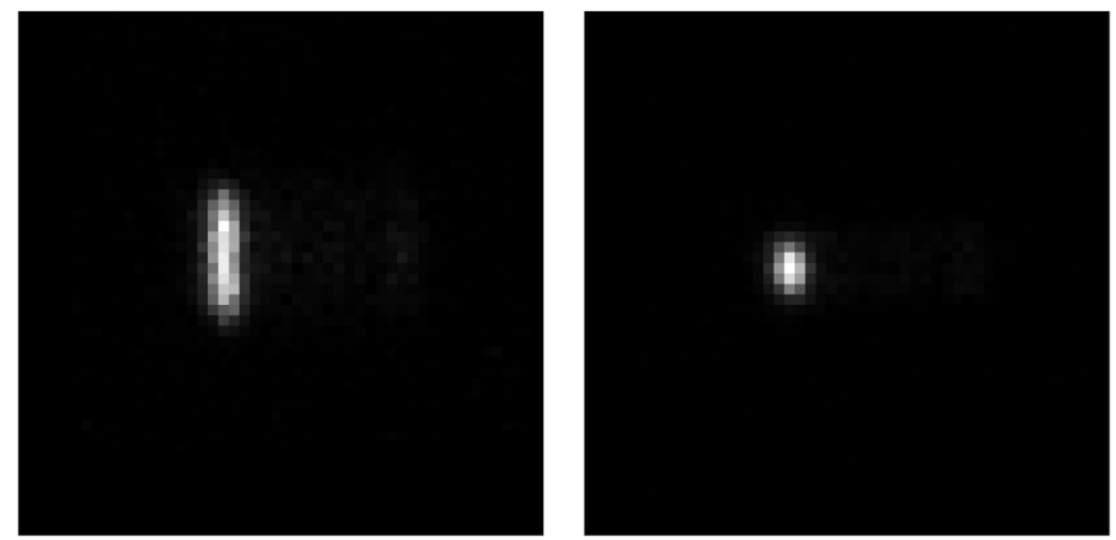

(a)
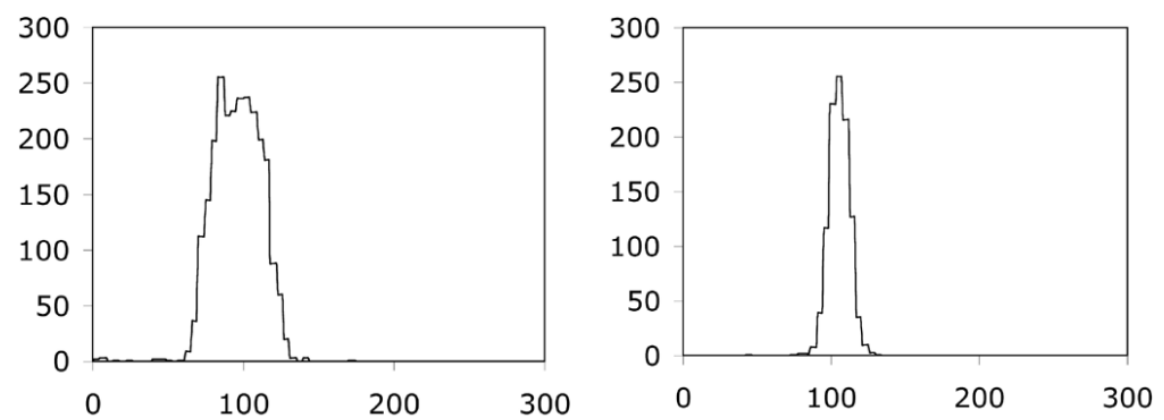

(b)

Fig. 6. (a) Planar images of Americium 241 point source "wiggled" while being imaged by the gamma cameras and the IR tracking system. (b) Profiles (in arbitrary units) are of a vertical line through the center of the source image.

\section{A. Larger FOV Detector Head Development}

Two $10 \mathrm{~cm} \times 20 \mathrm{~cm}$ SPECT gamma camera detector heads based on a $4 \times 8$ array of the Hamamatsu R8520-C12 $(2.5 \mathrm{~cm}$ $\times 2.5 \mathrm{~cm} ; 6 X \times 6 Y$ anodes) PSPMTs have been built and are being integrated into the gantry. Fig. 8 is a photograph of the array of PSPMTs mounted to the front-end electronics circuit board. 

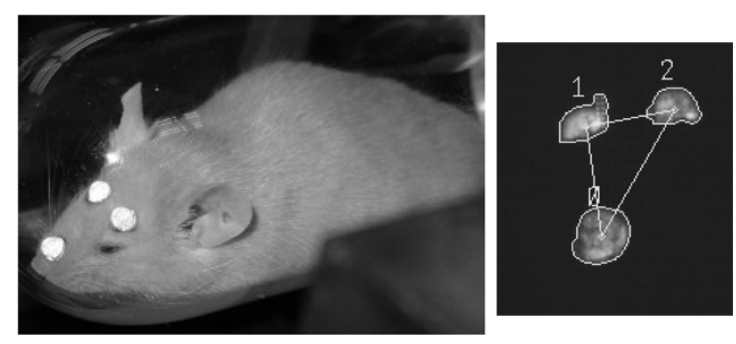

Fig. 7. (Left) photograph of the mouse with three IR hemispherical retroreflective markers attached to the head. (Right) Single frame from one of the two IR CMOS cameras showing the orientation of the set of three IR hemispherical retroreflective markers.

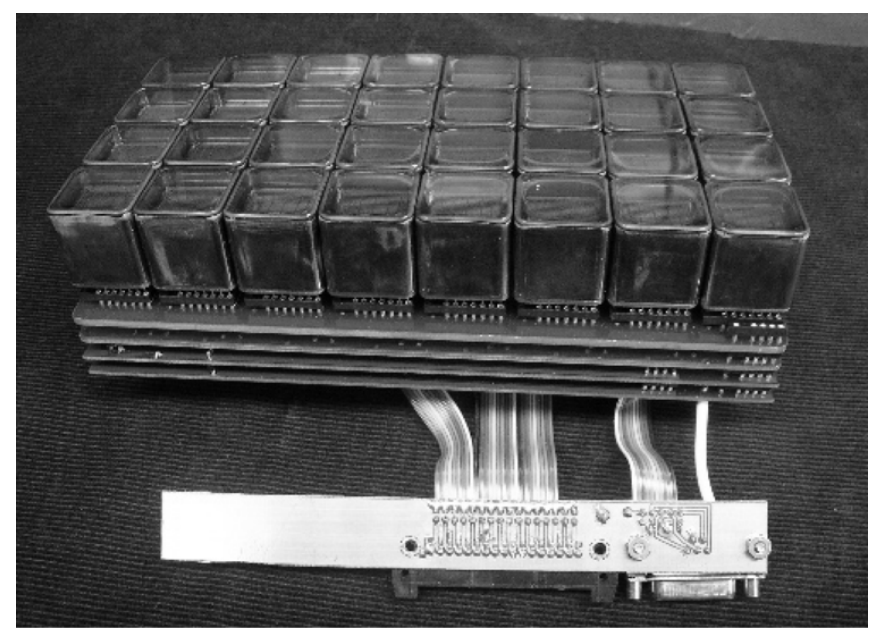

Fig. 8. Photograph of $10 \mathrm{~cm} \times 20 \mathrm{~cm}$ detector head based on a $4 \times 8$ array of R8520-C12 PSPMTs.

The readout circuitry for the 32 R8520-00-C12 PSPMTs, which make up the array of the PSPMTs, uses a similar subtractive technique as used with the R2487. The anode signals are fed into a resistive array. The resistive array consists of a linear chain of resistors on the $X$ and $Y$ coordinates resulting in 24 channels in the $X$ coordinate and 48 in the $Y$. The signal from this chain is picked off at the coordinate ends and between each PSPMT row or column to produce $5 X$ and $9 Y$ readout channels for each detector head. Please see Fig. 9 for a schematic of a portion of the readout circuit in which the interconnection and readout of two of the 32 PSPMTs is shown.

The array of PSPMTs is coupled to a $\mathrm{NaI}(\mathrm{Tl})$ array obtained from Saint Gobain in which the crystal elements are $2 \mathrm{~mm} \times 2$ $\mathrm{mm} \times 15 \mathrm{~mm}$ in size with a $0.25-\mathrm{mm}$ septa. The detectors extend axially so that the entire animal can be imaged on a $10-\mathrm{cm}-$ wide $\times 20$-cm-long scintillator array. The detector heads can be equipped with either parallel hole or pinhole collimators.

With the planned parallel hole collimators and the intrinsic position resolution of these detectors being limited by the $2.25-\mathrm{mm}$ step of the elements of the scintillator array, the expected position resolution will be on the order of 3-mm FWHM at a distance of $1 \mathrm{~cm}$ from the collimator face. The expected sensitivity will be $378 \mathrm{cpm} / \mu \mathrm{Ci}$. To facilitate the imaging of small features, we plan to use pinhole collimation. The highest resolution small animal SPECT studies have previously been reported to be accomplished with pinhole collimation [35]-[37]. Pinhole collimation permits high-resolution SPECT imaging of small animals close to the pinhole aperture. Sensitivity is generally more than an order of magnitude less than that for small animal PET, which has led

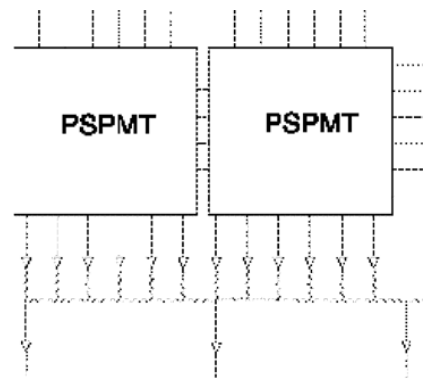

Fig. 9. Schematic of a section of the readout circuit showing two of the 32 PSPMTs to illustrate the manner in which the signals are picked off at coordinate ends and between PSPMTs.

to efforts to employ multipinhole collimation to improve count rates [38], [39]. The feasibility of multipinhole collimation with projection data overlap for the estimate of activity distribution in a small animal such as a mouse is currently not well understood.

In order to investigate the possibility of improving system sensitivity for pinhole collimators, we are investigating for our application multipinhole collimation with image overlap [40], [41]. We have described in [42] the design and construction of multipinhole collimators to image the mouse at different positions inside the burrow.

\section{B. Tracking Intrinsic Features}

We are also investigating using a triangulation technique called profilimetry [43] to track intrinsic features on the body of the mouse rather that externally attached markers. This is being investigated in an attempt to be able to track the position and pose of the torso. The profilimetry system consists of 1) two lasers (near infrared, $732 \mathrm{~nm}$ ) with diffractive optics that form the laser beam into a line and 2) the same two $512 \times 512$ CMOS element sensor cameras used for imaging the retroreflectors. The laser line is projected onto the moving object of interest while the object moves beneath the laser, normal to the laser line. The camera continuously images the object as the projected laser beam scans the object's surface. The triangulation algorithm, which extracts the object height based on the shape of the projected laser profile, is directly programmed into the CMOS sensor of the camera. This allows the height information to be extracted at a maximum rate of 2000 lines per second. The two laser/camera combination units are used to image opposite sides of the animal, resulting in a nearly complete surface of the whole animal.

\section{ACKNOWLEDGMENT}

The authors would like to thank Dr. E. Bradley of the College of William and Mary, Dr. J. Wall of the University of Tennessee Graduate School of Medicine, Dr. S. Kennel of the Oak Ridge National Laboratory, and Dr. J. Gregor of the University of Tennessee for valuable assistance with these studies. The authors would also like to thank Dr. C. Zorn for a thorough reading of our manuscript.

\section{REFERENCES}

[1] P. Pieri, A. J. Fischman, M. Ahmad, R. H. Moore, R. J. Callahan, and H. W. Strauss, "Cardiac blood-pool scintigraphy in rats and hamsters-Comparison of 5 radiopharmaceuticals and 3 pinhole collimator apertures," J. Nucl. Med., vol. 32, no. 5, pp. 851-855, 1991. 
[2] D. A. Weber, M. Ivanovic, D. Franceschi, S. E. Strand, K. Erlandsson, M. Franceschi, H. L. Atkins, J. A. Coderre, H. Susskind, T. Button, and K. Ljunggren, "Pinhole SPECT: An approach to in vivo high resolution SPECT imaging in small laboratory animals," J. Nucl. Med., vol. 35, no. 2, pp. 342-348, Feb. 1994

[3] R. J. Jaszczak, L. Jianying, H. Wang, M. R. Zalutsky, and R. E. Coleman, "Pinhole collimation for ultra-high-resolution, small-field-of-view SPECT," Phys. Med. Biol., vol. 39, no. 3, pp. 425-437, 1994.

[4] K. Ishizu, T. Mukai, Y. Yonekura, M. Pagani, T. Fujita, Y. Magata, S. Nishizawa, N. Tamaki, H. Shibasaki, and J. Konishi, "Ultra-high resolution SPECT system using four pinhole collimators for small animal studies," J. Nucl. Med., vol. 36, no. 12, pp. 2282-2287, Dec. 1995.

[5] F. A. Dilmanian, D. A. Weber, J. A. Coderre, D. D. Joel, K.-C. Shi, G. E. Meinken, P. Som, Y.-N. Tang, N. D. Volkow, C. Yee, A. B. Brill, M. Watanabe, E. Inuzuka, K. Oba, R. Gerson, H. Iida, and A. Hiruma, "A high-resolution SPECT system based on a microchannel-plate imager," IEEE Trans. Nucl. Sci., pt. 1, vol. 37, no. 2, pp. 687-695, Apr. 1990.

[6] A. G. Weisenberger, E. Bradley, S. Majewski, and M. Saha, "Development of a novel radiation imaging detector system for in vivo gene imaging in small animal studies," in Conf. Rec. 1996 IEEE Nuclear Science Symp. Medical Imaging Conf., Anaheim, CA, Nov. 2-9, 1996, pp. 1201-1205.

[7] R. Wojcik et al., "High spatial resolution gamma imaging detector based on a 5 " diameter R3292 Hamamatsu PSPMT," IEEE Trans. Nucl. Sci., vol. 45, no. 3, pp. 487-491, Jun. 1998.

[8] N. Schramm, A. Wirrwar, F. Sonnenberg, and H. Halling, "Compact high resolution detector for small animal SPECT," IEEE Trans. Nucl. Sci., vol. 47, no. 3, pp. 1163-1167, Jun. 2000.

[9] M. V. Green, J. Seidel, and W. R. Gandler, "A small animal system capable of PET, SPECT and planar imaging," J. Nuc. Med., vol. 35, no. 5, pp. P61-P61, 1994.

[10] L. R. MacDonald, B. E. Patt, J. S. Iwanczyk, B. M. W. Tsui, Y. Wang, E. C. Frey, D. E. Wessell, P. D. Acton, and H. F. Kung, "Pinhole SPECT of mice using the LumaGEM gamma camera," IEEE Trans. Nucl. Sci., vol. 48, no. 3, pp. 830-836, Jun. 2001.

[11] D. P. McElroy et al., "Performance evaluation of A-SPECT: A high resolution desktop pinhole SPECT system for imaging small animals," IEEE Trans. Nucl. Sci., vol. 49, no. 5, pp. 2139-2147, Oct. 2002.

[12] S. R. Cherry, Y. Shao, R. W. Silverman, K. Meadors, S. Siegal, A. Chatziioannou, J. W. Young, W. F. Jones, J. C. Moyers, D. Newport, A. Boutefnouchet, T. H. Farquhar, M. Andreaco, M. Paulus, D. M. Binkley, and R. Nutt, "MicroPET: A high resolution PET scanner for imaging small animals," IEEE Trans. Nucl. Sci., vol. 44, no. 3, pp. 1161-1166, Jun. 1997.

[13] K. Ishizu, T. Mukai, Y. Yonekura, M. Pagani, T. Fujita, Y. Magata, S. Nishizawa, N. Tamaki, H. Shibasaki, and J. Konishi, "Ultra-high resolution SPECT system using four pinhole collimators for small animal studies," J. Nucl. Med., vol. 36, no. 12, pp. 2282-2287, Dec. 1995.

[14] H. I. Kornblum, D. M., A. J. Annala, K. J. Tatsukawa, M. E. Phelps, and S. R. Cherry, "In vivo imaging of neuronal activation and plasticity in the rat brain by high resolution positron emission tomography (microPET)," Nature Biotechnol., vol. 18, pp. 655-660, 2000.

[15] P. D. Acton, S. Choi, K. Plössl, and H. F. Kung, "Quatification of dopamine transporters in the mouse brain using ultra-high resolution single-photon emission tomography," Eur. J. Nucl. Med., vol. 29, pp. 691-698, 2002.

[16] R. S. Balaban and V. A. Hampshire, "Challenges in small animal noninvasive imaging," ILAR J., vol. 42, no. 3, pp. 248-262, 2001.

[17] C. L. Woody, S. P. Stoll, D. J. Schlyer, M. Gerasimov, P. Vaska, S. Shokouhi, N. Volkow, J. S. Fowler, and S. L. Dewey, "A study of scintillation beta microprobes," in Conf. Rec. 2001 IEEE Nuclear Science Symp. Medical Imaging Conf., vol. 4, Nov. 2001, pp. 2002-2007.

[18] L. Zimmer, W. Hassoun, F. Pain, F. Bonnefoi, P. Laniece, R. Mastrippolito, L. Pinot, J. F. Pujol, and V. Leviel, "SIC, an intracerebral beta (+)-range-sensitive probe for radiopharmacology investigations in small laboratory animals: Binding studies with (11)C-raclopride," $J$. Nucl. Med., vol. 43, no. 2, pp. 227-233, Feb. 2002.

[19] P. Vaska, D. J. Schlyer, C. L. Woody, S. P. Stoll, V. Radeka, and N. Volkow, "Imaging the unanesthetized rat brain with PET: A feasibility study," in Conf. Rec. 2001 IEEE Nuclear Science Symp. Medical Imaging Conf., vol. 3, Nov. 2001, pp. 1569-1571.

[20] M.J.Paulus, S. S. Gleason, S.J.Kennel, P.R. Hunsicker, andD.K. Johnson, "High resolution x-ray computed tomography: An emerging tool for small animal cancer research," Neoplasia, vol. 2, pp. 62-70, 2000.

[21] M. J. Paulus, H. Sari-Sarraf, S. S. Gleason, M. Bobrek, J. S. Hicks, D. K. Johnson, J. K. Behel, L. H. Thompson, and W. C. Allen, "A new X-ray computed tomography system for laboratory mouse imaging," IEEE Trans. Nucl. Sci., vol. 46, no. 3, pp. 558-564, Jun. 1999.

[22] S. S. Gleason, H. Sari-Sarraf, M. J. Paulus, D. K. Johnson, S. J. Norton, and M. A. Abidi, "Reconstruction of multi-energy, X-ray computed tomography images of laboratory mice," IEEE Trans. Nucl. Sci., vol. 46, no. 4, pp. 1081-1086, Aug. 1999.
[23] E. Bradley, private communication, 2002 Biology Department, College of William and Mary, Williamsburgh, VA.

[24] R. Wojcik, S. Majewski, B. Kross, V. Popov, and A. G. Weisenberger, "Optimized readout of small gamma cameras for high resolution single gamma and positron emission imaging," in Conf. Rec. 2001 IEEE Nuclear Science Symp. Medical Imaging Conf., vol. 3, Nov. 2001, pp. $1821-1825$.

[25] B. L. Welch, S. Majewski, V. Popov, A. G. Weisenberger, R. Wojcik, J. S. Goddard, S. S. Gleason, and M. J. Paulus, "Acquisition and control for a combined SPECT/infrared tracking system for restraint-free small animal imaging," in Conf. Rec. 2003 IEEE Nuclear Science Symp. Medical Imaging Conf., vol. 4, Oct. 2003, pp. 2310-2314.

[26] R. R. Fulton, S. R. Meikle, S. Eberl, J. Pfeiffer, C. J. Constable, and M. J. Fulham, "Correction for head movements in positron emission tomography using an optical motion-tracking system," IEEE Trans. Nucl. Sci, vol. 49, no. 1, pp. 116-123, Feb. 2002.

[27] P. M. Bloomfield, T. J. Spinks, J. Reed, L. Schnorr, A. M. Westrip, L. Livieratos, R. Fulton, and T. Jones, "The design and implementation of a motion correction scheme for neurological PET," Phys. Med. Biol., vol. 48, no. 8, pp. 959-978, 2003.

[28] M. A. Gennert, P. P. Bruyant, M. V. Narayanan, and M. A. King, "Assessing a system to detect patient motion in SPECT imaging using stereo optical cameras," in Conf. Rec. 2001 IEEE Nuclear Science Symp. Medical Imaging Conf., vol. 3, 2003, pp. 1567-1570.

[29] R. R. Fulton, S. Eberl, S. R. Meikle, B. F. Hutton, and M. Braun, "A practical 3D tomographic method for correcting patient head motion in clinical SPECT," IEEE Trans. Nucl. Sci., vol. 46, no. 3, pp. 667-672, Jun. 1999.

[30] E. Gravier and Y. Yang, "Motion-compensated reconstruction of tomographic image sequences," in Proc. IEEE Int. Conf. Image Processing, vol. 2, 2003, pp. 807-810.

[31] M. Ivanovic, D. A. Weber, S. Loncaric, C. Pellot-Barakat, and D. K. Shelton, "Patient motion correction for multicamera SPECT using $360^{\circ}$ acquisition/detector," in Con. Rec. 1997 IEEE Nuclear Science Symp. Medical Imaging Conf., vol. 2, 1997, pp. 989-993.

[32] A. Z. Kyme, B. F. Hutton, R. L. Hatton, D. W. Skerrett, and L. R. Barnden, "Practical aspects of a data-driven motion correction approach for brain SPECT," IEEE Trans. Med. Imag., vol. 22, no. 6, pp. 722-729, Jun. 2003.

[33] J. S. Goddard, S. S. Gleason, M. J. Paulus, A. Weisenberger, M. Smith, B. Welch, and R. Wojcik, "Real-time landmark-based unrestrained animal tracking system for motion-corrected PET/SPECT imaging," in Conf. Rec. 2002 IEEE Nuclear Science Symp. Medical Imaging Conf., vol. 3, Nov. 2002, pp. 1534-1537.

[34] Z. Zhang, "A flexible new technique for camera calibration," Microsoft Research, Microsoft Corp., Redmond, WA, MSR-TR-98-71, 1998.

[35] D. A. Weber, M. Ivanovic, D. Franceschi, S.-E. Strand, K. Erlandsson, M. Franceschi, H. L. Atkins, J. A. Coderre, H. Susskind, T. Button, and K. Ljunggren, "Pinhole SPECT: An approach to in vivo high resolution SPECT imaging in small laboratory animals," J. Nucl. Med., vol. 35, pp. 342-348, 1994.

[36] R. J. Jaszczak, J. Li, H. Wang, M. R. Zalutsky, and R. E. Coleman, "Pinhole collimation for ultra-high-resolution, small-field-of-view SPECT," Phys. Med. Biol., vol. 39, pp. 425-437, 1994.

[37] M. C. Wu, H. R. Tang, D. W. Gao, A. Ido, J. W. O'Connell, B. H. Hasegawa, and M. W. Dae, "ECG-gated pinhole SPECT in mice with millimeter spatial resolution," IEEE Trans. Nucl. Sci., vol. 47, no. 3, pp. 1218-1221, Jun. 2000.

[38] N. U. Schramm, G. Ebel, U. Engeland, M. Schurrat, M. Béhé, and T. M. Behr, "High resolution SPECT using multi-pinhole collimation," IEEE Trans. Nucl. Sci., pt. 2, vol. 50, no. 3, pp. 315-320, Jun. 2003.

[39] S. R. Meikle, R. R. Fulton, S. Eberl, M. Dahlbom, K.-P. Wong, and M. J. Fulham, "An investigation of coded aperture imaging for small animal SPECT," IEEE Trans. Nucl. Sci., vol. 48, no. 3, pp. 816-821, Jun. 2001.

[40] M. F. Smith, S. R. Meikle, S. Majewski, and A. G. Weisenberger, "Design of multipinhole collimators for small animal SPECT," in Conf. Rec. 2003 IEEE Nuclear Science Symp. Medical Imaging Conf., vol. 4, Oct. 2003, pp. 2291-2295.

[41] S. R. Meikle, P. Kench, R. Wojcik, M. F. Smith, A. G. Weisenberger, S. Majewski, M. L. F. Lerch, and A. B. Rozenfeld, "Performance evaluation of a multipinhole small animal SPECT system," in Conf. Rec. 2003 IEEE Nuclear Science Symp. Medical Imaging Conf., vol. 3, Oct. 2003, pp. 1988-1992.

[42] M. F. Smith, S. Majewski, S. R. Meikle, A. G. Weisenberger, V. Popov, and R. F. Wojcik, "Design of high sensitivity, high resolution compact single photon imaging devices for small animal and dedicated breast imaging," in Conf. Rec. 2001 IEEE Nuclear Science Symp. Medical Imaging Conf., vol. 3, Nov. 2002, pp. 1592-1596.

[43] Laser Triangulation. [Online]. Available: http://www.ivp.se/documentation/technology/LaserTriangulation.pdf 